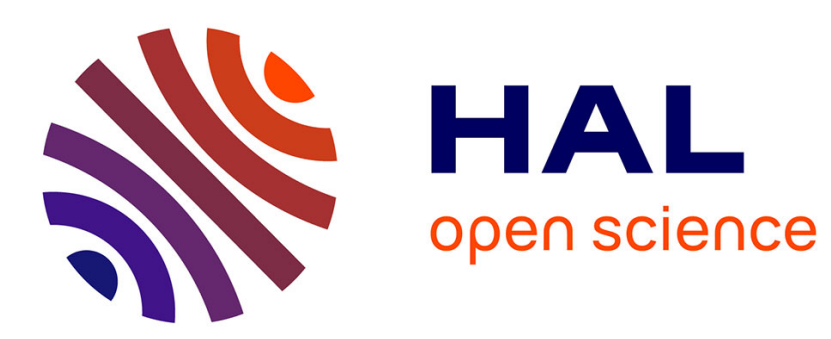

\title{
A Performance Metric for Co-optimization of Day-Ahead Dispatch and Reserves in Electric Microgrids
}

Mayank Panwar, Siddharth Suryanarayanan, Robin Roche, Rob Hovsapian

\section{To cite this version:}

Mayank Panwar, Siddharth Suryanarayanan, Robin Roche, Rob Hovsapian. A Performance Metric for Co-optimization of Day-Ahead Dispatch and Reserves in Electric Microgrids. PowerTech, Jun 2019, Milano, Italy. hal-02867751

\section{HAL Id: hal-02867751 https://hal.science/hal-02867751}

Submitted on 15 Jun 2020

HAL is a multi-disciplinary open access archive for the deposit and dissemination of scientific research documents, whether they are published or not. The documents may come from teaching and research institutions in France or abroad, or from public or private research centers.
L'archive ouverte pluridisciplinaire HAL, est destinée au dépôt et à la diffusion de documents scientifiques de niveau recherche, publiés ou non, émanant des établissements d'enseignement et de recherche français ou étrangers, des laboratoires publics ou privés. 


\title{
A Performance Metric for Co-Optimization of Day-Ahead Dispatch and Reserves in Electric Microgrids
}

\author{
Mayank Panwar ${ }^{1}$, Member, IEEE, Siddharth Suryanarayanan ${ }^{2}$, Senior Member, IEEE, \\ Robin Roche ${ }^{3}$, Senior Member, IEEE, and Rob Hovsapian ${ }^{1}$, Senior Member, IEEE.
}

\begin{abstract}
We present a new performance metric for cooptimization of dispatch and reserves in microgrids. A metric based on NERC criteria is used for each asset to quantify its reliability-based value. A system level metric is obtained through the individual performance metrics, dispatched capacity, and net dispatchable capacity available as reserve. Simulations are performed on a notional microgrid with a dispatchable and a non-dispatchable distributed energy resource to demonstrate the calculation of the metric.
\end{abstract}

Index Terms-dispatch; distributed energy resources; microgrids; reserves.

\section{INTRODUCTION}

Electric microgrids consist of DERs that have inherently different requirements of dispatchability than traditional generators in the EPS. Performance of traditional electric power grid generators is measured using NERC metrics. Some of the NERC metrics can be used to quantify the performance of dispatchable and non-dispatchable DERs in the microgrid [1]. However, the variability in the electric power output of non-dispatchable DERs and the inability to accurately forecast the output pose risks to power system scheduling and dispatch. Capacity reserves in the microgrid are a vital tool to mitigate this risk. Reserves management in the traditional EPS is done heuristically, such as allocating a constant (fixed) percentage of generation, based on load demand [2]. Traditional dispatch and reserve management do not consider performance as a criteria for operation. Therefore, the concept of using performance metrics in the operation of microgrids is proposed here. Further, reserve management in the grid-connected mode of operation of the microgrid is chosen as an area of application of performance metrics.

The focus of this work is on the grid-connected mode of operation of the microgrid, as the microgrid is expected to operate in this mode for majority of the time. Dispatch can also include peak load reduction, emissions, reliability, and other operator-defined criterion. Under such cases, reserve management can be even more challenging. Therefore, a

This work was supported and funded through a joint collaborative research initiative between Colorado State University, Fort Collins, CO, USA, and Idaho National Laboratory, Idaho Falls, ID, USA (contract number 5-300722).

\footnotetext{
${ }^{1}$ M. Panwar and R. Hovsapian are with Idaho National Laboratory, Idaho Falls, ID, USA (emails:Mayank.Panwar@INL.Gov and Rob.Hovsapian@INL.Gov)

${ }^{2} \mathrm{~S}$. Suryanarayanan is with Colorado State University, Fort Collins, CO, USA (email: Sid.Suryanarayanan@Colostate.Edu)

${ }^{3} \mathrm{R}$. Roche is with FEMTO-ST, CNRS, Univ. Bourgogne Franche-Comte, UTBM, Belfort, France (email: Robin.Roche@UBFC.Fr)
}

systematic approach is required to capture the performance capability of the microgrid to mitigate the risk posed by the inherent variability of non-dispatchable DER during dispatch and scheduling. Instead of relying completely on the EPS to mitigate this risk, the concept of self-provision of reserves, locally from the microgrid DERs, is explored here.

We apply a NERC metric, namely the NOF, to quantify reliability of microgrid assets and incorporate these with other factors in the microgrid such as load demand, capacity rating, and power output of the non-dispatchable and dispatchable assets. All of the above-mentioned factors are used to formulate a performance metric, named the $\mathcal{R}$-metric, to quantify the net value of reserve of the microgrid through self-provision. The characteristics of the constituent DERs are measured through traditional metrics and combined with the new performance metric to evaluate the reliability-based cost and value of the unused reserves of the microgrid. The reliability-based metrics for reserve are then used as a constraint in the dispatch. This performance metric, $\mathcal{R}$, is applied for reserve management in day-ahead dispatch. The advantage of using the new developed metric, $\mathcal{R}$, is that the reserve constraint considers the reliability and performance measures that are indicative of the quality of the constituent assets in a microgrid, both dispatchable and nondispatchable.

The rest of the paper is organized as follows: section II explains the reliability evaluation of reserves; section III describes the application of the development of the new performance metric, $\mathrm{R}$, and its application to reserve management in day-ahead dispatch; section IV presents the data and models used in the ED problem; section V details the results along with a sensitivity analysis in section VI; section VII concludes.

\section{Reliability-based Evaluation of Reserve}

Firstly, we define the quantities used in formulation of the metrics. These are shown for an example load demand for one time period in Figure 1.

NOF is defined as the ratio of net actual generation to the product of net maximum capacity and the sum of all unit service hours [3]. The NOF for the dispatchable and non-dispatchable assets in the microgrid DERs are given by (1) and (2), respectively. Typical values of NOF for the EPS, dispatchable DER, and non-dispatchable DER are 81.5, 52.1, and 40.3 , respectively [3], [4]. Reliability is quantified for the dispatched power and dispatchable reserve. Some of the metrics, previously developed in [1], are used here. These are 


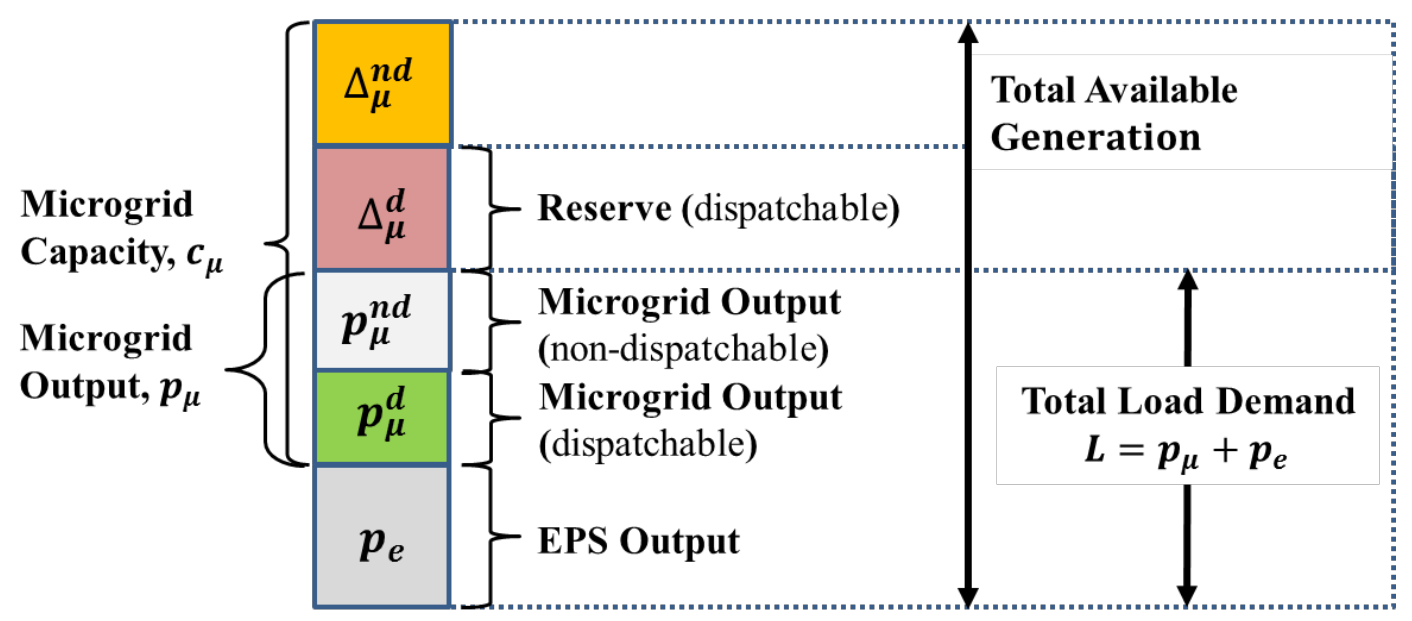

Fig. 1. System level representation of various capacity-based quantities for one time period.

the peak reserve ratio, $\operatorname{prr}(t)$, given in (3), and the peak load reduction, $p l r(t)$, given in (4). Peak reserve ratio is defined as the ratio of the microgrid reserve capacity to the total feeder load. Peak load reduction is defined as the ratio of the peak load with DERs to the peak load without DERs [4].

$$
\begin{gathered}
N O F_{d}=\frac{\sum_{i=1}^{t_{\text {period }}} p_{\mu}^{d} t_{i}}{\left(c_{\mu}^{d} t_{\text {period }}\right)} \times 100 \% \\
N O F_{n d}=\frac{\sum_{i=1}^{t_{\text {period }}} p_{\mu}^{n d} t_{i}}{\left(c_{\mu}^{n d} t_{\text {period }}\right)} \times 100 \% \\
\operatorname{prr}(t)=\frac{\Delta \mu(t)}{p_{e}(t)+p_{\mu}(t)} \\
\operatorname{plr}(t)=\frac{p_{\mu}(t)}{p_{e}(t)+p_{\mu}(t)}
\end{gathered}
$$

where $t$ represents the time index.

Using the reserve quantities on a load demand curve, we can calculate the value and cost of microgrid reserve as seen by the DSO. The reliability value of unused reserve, rvur, given by (5), signifies the minimum acceptable level of reliability-weighted capacity measure for equivalent reserve. Value is derived as the product of microgrid reserve capacity and weighted reliability of the dispatched generation from EPS and microgrid. The reliability cost of unused reserve, rcur, given by (6), signifies the reliability-weighted cost of using the full capacity of microgrid, i.e., a scenario corresponding to zero-reserve.

$$
\begin{aligned}
\operatorname{rvur}(t) & =\Delta_{\mu}^{d} \frac{p_{e} w_{e}+p_{\mu} w_{\mu}}{p_{e}+p_{\mu}}=\operatorname{prr}^{d}\left(p_{e} w_{e}+p_{\mu} w_{\mu}\right) \\
\operatorname{rcur}(t) & =\Delta_{\mu}^{d} \frac{\left(p_{e}-\Delta_{\mu}^{d}\right) w_{e}+p_{\mu} w_{\mu}+\Delta_{\mu}^{d} w_{\Delta_{\mu}}^{d}}{p_{e}+p_{\mu}} \\
& =\operatorname{prr}^{d}\left(\left(p_{e}-\Delta_{\mu}^{d}\right) w_{e}+p_{\mu} w_{\mu}+\Delta_{\mu}^{d} w_{\Delta_{\mu}}^{d}\right)
\end{aligned}
$$

where $p^{d} r^{d}=\frac{\Delta_{\mu}^{d}}{p_{e}+p_{\mu}}, p_{\mu} w_{\mu}=p_{\mu}^{d} w_{\mu}^{d}+p_{\mu}^{n d} w_{\mu}^{n d}$, and $\Delta_{\mu}=\Delta_{\mu}^{d}+\Delta_{\mu}^{n d}$. Note that we use the NOF as a proxy for $w_{e}$ and $w_{\mu}$ in (5)-(10). It may be noted that $\operatorname{prr}^{d}$ and $\Delta_{\mu}^{d}$ correspond to the dispatchable DERs in the microgrid only, and is different from (3), which considers all the dispatchable and non-dispatchable DERs.

Note that both rvur and rcur are given in units of electric power. Cost of reserve is derived by product of microgrid reserve capacity and weighted reliability of the generation serving the load when full capacity of microgrid is dispatched leaving no reserve from microgrid. Ideally, the weighted reliability metric can be $\leq 1.0$.

Figure 2 shows the relationship of the above-mentioned metrics for a typical grid-connected operation mode of a microgrid.

\section{Application to Reserve Management in DAY-AHEAD DISPATCH}

Reserve management is non-trivial in a heterogeneous microgrid due to the varying dispatchability associated with the constituent DERs. Traditionally, reserve is calculated based on economic and capacity criteria [2]. We propose an alternative method to calculate the reliability-based value of capacity reserve by using the NOF, the rvur, and the rcur [5].

Here, both the EPS and the microgrid assets serve the constituent loads in the microgrid. Energy management decisions to allocate supply to the loads will occur in a typical ED considering operational costs. In the islanded mode operation of the microgrid, an appropriate reference reliability measure 


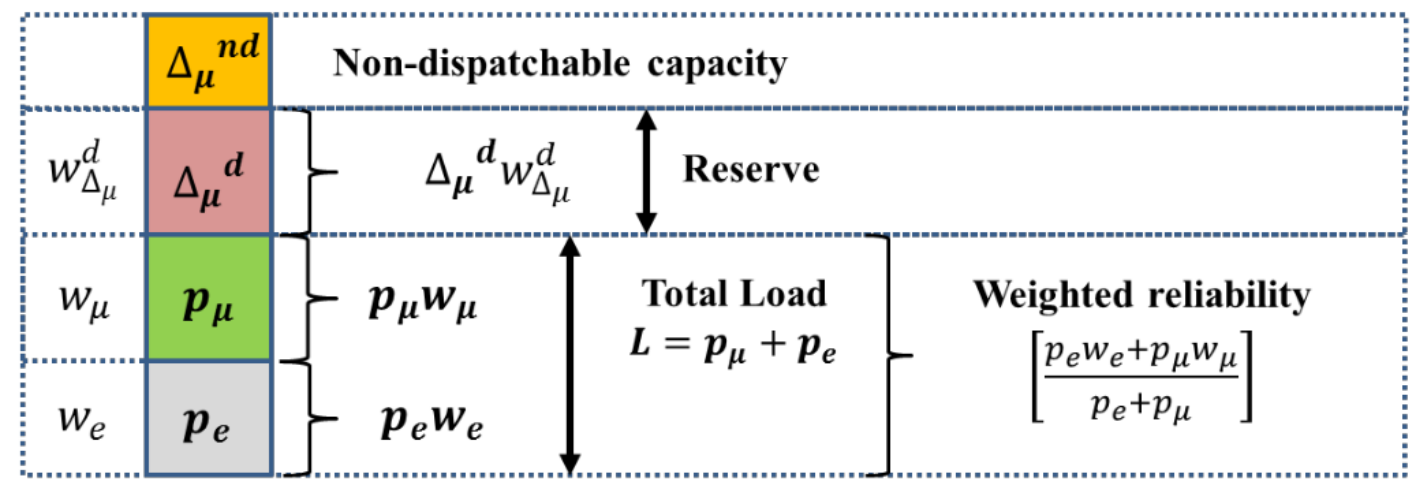

Fig. 2. A bar graph for microgrid operation with microgrid capacity reserve.

can be assigned instead of $w_{e}$, and applied similar to the grid-connected mode.

For the grid-connected mode, we calculate the change in net value of reliability that can occur by fully utilizing all the microgrid assets before relying on the EPS. This is given in (7) as the difference of (5) and (6).

$$
\mathcal{R}=\operatorname{prr}^{d}\left(\Delta_{\mu}^{d} w_{e}-\Delta_{\mu}^{d} w_{\Delta_{\mu}}^{d}\right)
$$

From Figure 1 we have $c_{\mu}^{d}=p_{\mu}^{d}+\Delta_{\mu}^{d}$, and $L=p_{\mu}+p_{e}$; by substituting in (7) and rearranging the terms, we get

$$
\mathcal{R}=\frac{\left(c_{\mu}^{d}-p_{\mu}^{d}\right)^{2}\left(w_{e}-w_{\Delta_{\mu}}^{d}\right)}{L}
$$

Extrema of $\mathcal{R}$ provide the lower and upper bounds on this metric. For a minimum capacity reserve assigned heuristically, say $\Delta_{\min }$, and assuming $\left(w_{e}-w_{\Delta_{\mu}}^{d}>0\right)$, the minimum and maximum values of $\mathcal{R}$ are obtained from (9) and (10), respectively.

$$
\begin{aligned}
& \min (\mathcal{R})=\frac{\left(\Delta_{\text {min }}\right)^{2}\left(w_{e}-w_{\Delta_{\mu}}^{d}\right)}{L} \\
& \max (\mathcal{R})=\frac{\left(c_{\mu}^{d}\right)^{2}\left(w_{e}-w_{\Delta_{\mu}}^{d}\right)}{L}
\end{aligned}
$$

We use a threshold of $20 \%$ reserve capacity in simulations presented in this paper to account for the error in forecasting solar PV outputs; this is a typical rule-of-thumb in EPS [6].

\section{Simulation Data AND ED}

Simulations are performed on a notional microgrid, from [7], with a diesel generator (dispatchable) and a solar PV DER (non-dispatchable), of $200 \mathrm{~kW}$ rating each. The simulation data used in this study are taken directly from [7]. The ED problem is formulated as (11)-(14).

$$
\begin{array}{r}
\min \left(f_{\mu}^{d} p_{\mu}^{d}+\text { TOU } p_{e}\right) \\
\text { s.t. }-\Delta_{\mu}^{d} \leq-\Delta_{\min } \\
0 \leq p_{\mu}^{d} \leq c_{\mu}^{d} \\
p_{\mu}+p_{e}=L
\end{array}
$$

Power-flow constraints are not considered, but can be included without loss of generality. The cost function of the dispatchable diesel DER, $f_{\mu}^{d}$, shown in (15), is derived from [8], [9] by using the generator unit parameters corresponding to the $200 \mathrm{~kW}$ diesel generator (from Table 1 of [8]), the diesel consumption rate $(14.4 \mathrm{gal} / \mathrm{hr})$, and the cost of diesel apropos to the time considered for the case study $(\$ 2.77 / \mathrm{gal}$ for the Rocky Mountain region) taken from [9]. Detailed information on this can be obtained from [7], [5].

$$
f_{\mu}^{d}=7.5992\left(\frac{p_{\mu}^{d}}{c_{\mu}^{d}}\right)^{2}+26.0886\left(\frac{p_{\mu}^{d}}{c_{\mu}^{d}}\right)+6.3506 .
$$

The ToU price for the period considered in this case study is $0.2202 \$ / \mathrm{kWh}$ for the $14^{\text {th }}$ to $19^{\text {th }}$ hour, and $0.0624 \$ / \mathrm{kWh}$ otherwise for the EPS [10] — note that these rates may be historical and may have changed. The load demand curve is obtained from [11] and linearly scaled by a factor of $10^{-3}$ (from MW to $\mathrm{kW}$ values). Solar irradiance and temperature data are taken directly from [12]. The simulation data are shown in Table I.

The value of $p_{\mu}^{n d}$ in Table I is calculated as shown in (16) using $S_{i r r}$ from [12]; $S_{\text {stm }}=1000 \mathrm{~W} / \mathrm{m}^{2} ; k=-0.0046$; $T_{r}=25{ }^{\circ} \mathrm{C}$; and $T_{c}$ from [7], [13].

$$
p_{\mu}^{n d}=c_{\mu}^{n d} \frac{S_{i r r}}{S_{s t m}}\left(1+k\left(T_{c}-T_{r}\right)\right)
$$

Table II shows the average hourly cost for various cases of capacity reserves maintained during the ED. The EPS-only case represents no use of DERs and has an average hourly cost of $\$ 42.68$. The values of $\mathcal{R}$ and the weighted reliability metrics are calculated after executing the ED. This shows the behavior of the $\mathcal{R}$-metric in a classical ED setup.

Case-1 is a classical ED problem without any reserve constraint. Cases 2-4 represent reserve constraints as $20 \%$ of $c_{\mu}^{d}, L$, and $p_{\mu}^{n d}$, respectively, and are the base cases for comparison with the ED using $\mathcal{R}$-metric constraint. The weighted reliability of the reserve is same in all cases due to the presence of a single dispatchable DER as shown in Table II. This is a capacity weighted reliability quantity and can change in case of multiple dispatchable DERs. Table III 
TABLE I

DATA FOR LOAD DEMAND AND SOLAR OUTPUT

\begin{tabular}{|c|c|c|}
\hline Hour $(\mathrm{h})$ & $L(\mathrm{~kW})$ & $p_{\mu}^{n d}$ \\
\hline 1 & 328 & 0 \\
2 & 310 & 0 \\
3 & 299 & 0 \\
4 & 285 & 0 \\
5 & 296 & 0 \\
6 & 307 & 0 \\
7 & 330 & 3.89 \\
8 & 350 & 40.97 \\
9 & 371 & 79.31 \\
10 & 392 & 123.71 \\
11 & 413 & 155.32 \\
12 & 433 & 179.49 \\
13 & 445 & 191.98 \\
14 & 453 & 187.29 \\
15 & 462 & 179.66 \\
16 & 468 & 154.20 \\
17 & 477 & 121.02 \\
18 & 477 & 81.15 \\
19 & 458 & 36.79 \\
20 & 436 & 4.87 \\
21 & 430 & 0 \\
22 & 413 & 0 \\
23 & 376 & 0 \\
24 & 340 & 0 \\
\hline & & \\
\hline
\end{tabular}

TABLE II

ED WITH VARIOUS CAPACITY RESERVE CONSTRAINTS

\begin{tabular}{|c|c|c|c|c|c}
\hline \multirow{2}{*}{ Case } & \multirow{2}{*}{$\Delta_{\min }$} & \multirow{2}{*}{ ED $(\$ / \mathrm{h})$} & $\mathcal{R}(\mathrm{kW})$ & \multicolumn{2}{|c}{ Weighted reliability (p.u.) } \\
\cline { 5 - 6 } & & & & Dispatch & Reserve \\
\hline 1 & No constraint & 37.45 & 78.33 & 0.77 & - \\
2 & $20 \%$ of $c_{\mu}^{d}$ & 37.66 & 79.32 & 0.78 & 0.52 \\
3 & $20 \%$ of $L$ & 38.18 & 83.18 & 0.79 & 0.52 \\
4 & $20 \%$ of $p_{\mu}^{n d}$ & 37.57 & 78.85 & 0.78 & 0.52 \\
\hline
\end{tabular}

shows the results of the ED with $\mathcal{R}$-metric as the left-hand side of the constraint (12) as in cases $2-4$. So, constraint (12) can be modified as (17).

$$
-\mathcal{R} \leq-\Delta_{\min }
$$

TABLE III

ED WITH $\mathcal{R}$-METRIC AS LEFT-HAND SIDE OF RESERVE CONSTRAINT (17)

\begin{tabular}{|c|c|c|c|c|c|}
\hline \multirow{2}{*}{ Case } & \multirow{2}{*}{$\Delta_{\min }$} & \multirow{2}{*}{ ED $(\$ / \mathrm{h})$} & \multirow{2}{*}{$(\mathrm{kW})$} & \multicolumn{2}{|c|}{ Weighted reliability (p.u.) } \\
\cline { 5 - 6 } & & & Dispatch & Reserve \\
\hline 5 & $20 \%$ of $c_{\mu}^{d}$ & 38.68 & 88.33 & 0.80 & 0.52 \\
6 & $20 \%$ of $L$ & 39.85 & 101.35 & 0.81 & 0.52 \\
7 & $20 \%$ of $p_{\mu}^{n d}$ & 38.25 & 84.66 & 0.79 & 0.52 \\
\hline
\end{tabular}

\section{Results}

A higher value of $\mathcal{R}$ is desirable, and the net value indicates the potential equivalent capacity available between the load serving capacity and the dispatchable reserve. Case-4 and Case-7 represent the most practical microgrid constraint scenarios, where the reserve corresponds to the risk posed by the variability of non-dispatchable DER [6]. The 24-hour dispatch for Case-7 is shown in Figure 3 and the variation of $\mathcal{R}$-metric and risk capacity for (17) is shown in Figure 4. The

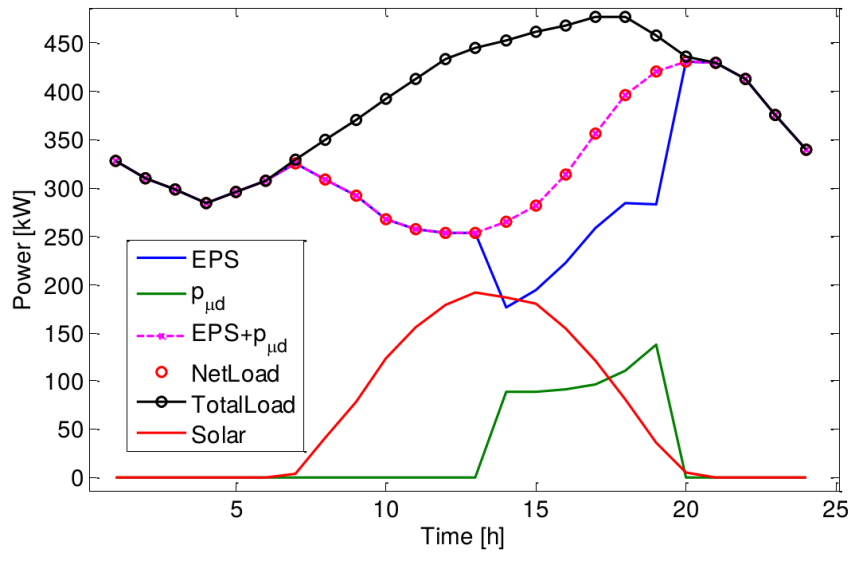

Fig. 3. Dispatch for Case-7 using $\mathcal{R}$-metric as reserve constraint.

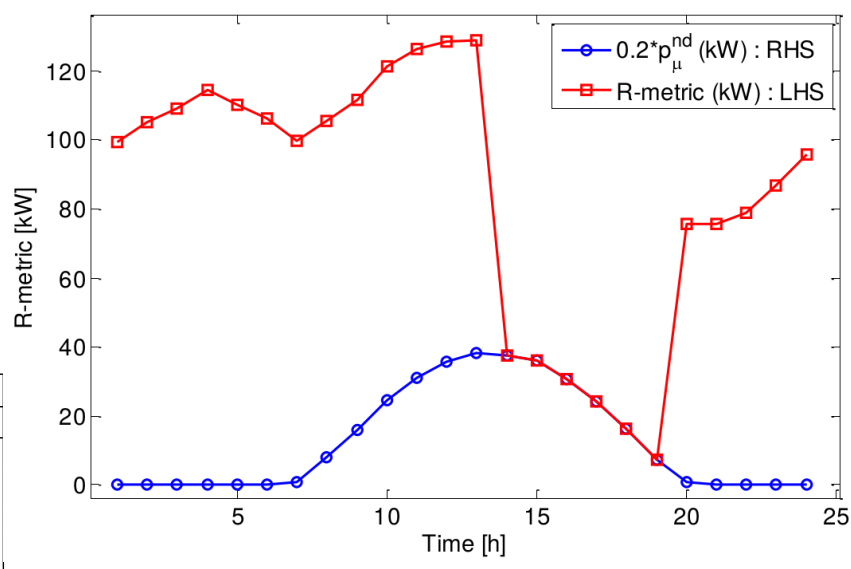

Fig. 4. Variation of $\mathcal{R}$-metric as reserve constraint in Case-7.

average power output, reserve, and reserve increase for the 24-hour period under consideration are shown in Table IV.

TABLE IV

COMPARISON OF AVERAGE DISPATCH AND RESERVE VALUES

\begin{tabular}{|c|c|c|c|c|}
\hline Case & $p_{e}(\mathrm{~kW})$ & $p_{\mu}^{d}(\mathrm{~kW})$ & $\Delta_{\mu}^{d}(\mathrm{~kW})$ & $\%$ increase in $\left(\Delta_{\mu}^{d}\right.$, cost $)$ \\
\hline 2 & 285.39 & 40.00 & 160.00 & $13.84,2.71$ \\
5 & 307.53 & 17.86 & 182.14 & $14.73,4.36$ \\
\hline 3 & 298.68 & 26.71 & 173.29 & $11.52,1.80$ \\
\hline 6 & 324.20 & 1.19 & 198.81 & 156.33 \\
\hline 7 & 281.72 & 43.67 & 174.35 & \\
\hline
\end{tabular}

The average dispatched capacity from the microgrid DERs is lower and the scheduled reserve is higher in cases 5-7 compared to cases 2-4. The value of dispatch reliability is also higher, which is attained by importing more power from the area EPS, and hence the higher cost of operation. Since the reserve is provided by the assets local to the microgrid, the EPS has an additional available capacity equal to the risk, i.e., $\Delta_{\min }=0.2 \times p_{\mu}^{n d}$. The average value of this additional capacity over 24-hours is $12.8303 \mathrm{~kW}$.

\section{Sensitivity AnAlysis}

Sensitivity analysis is performed through ED simulations by varying the NOF for EPS, dispatchable DER, PV, and 


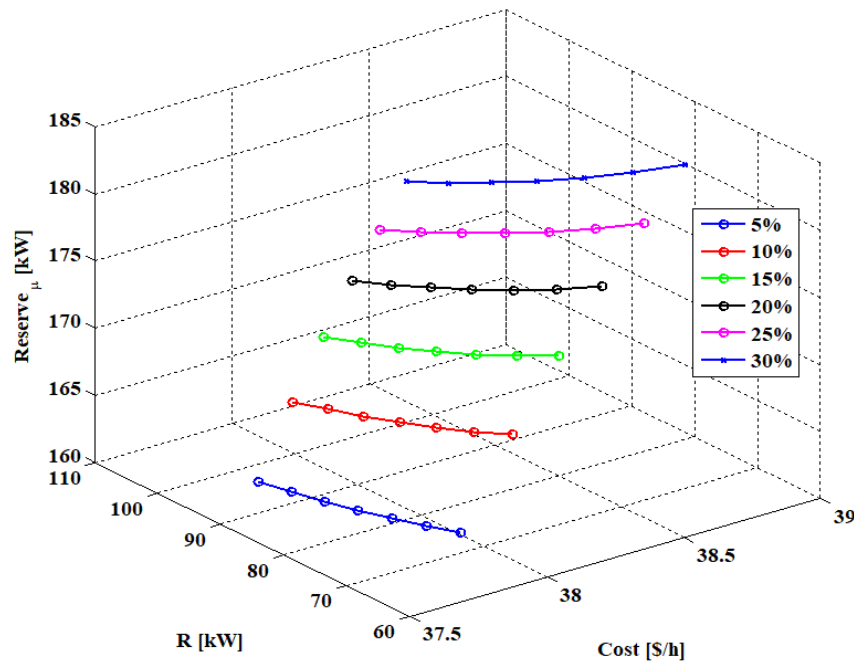

Fig. 5. Sensitivity analysis for $\mathcal{R}$-metric.

$\Delta_{\text {min }}$, i.e., the right-hand side of (17). The range for variations is based on typical values of each asset and heuristics [1], [3]-[6]. The range of variation of the NOF for the: EPS is [65\%, 95\%]; diesel generator is $[35 \%, 65 \%]$; and $\mathrm{PV}$ is [35\%, 55\%]. The variation in $\Delta_{\min }$ is $[5 \%, 30 \%]$ of $p_{\mu}^{n d}$ in steps of $5 \%$.

The results are plotted for the 24-hour average for operating cost, $\mathcal{R}$-metric, and $\Delta_{\mu}^{d}$ using different $\Delta_{\min }$, and are shown in Figure 5. It can be observed that for a particular risk in (17), a higher cost results in a higher reserve capacity, but a lower $\mathcal{R}$-metric value. This trend is consistent in all cases with varying magnitudes of variation. Thus, it is observed that the $\mathcal{R}$-metric has an inverse dependence with cost and the inverse effect increases (higher slope) as $\Delta_{\min }$ increases. The dispatchable reserve also has an inverse relationship with $\mathcal{R}$-metric. Therefore, a higher value of desired $\mathcal{R}$-metric will result in lower reserve $\Delta_{\mu}^{d}$. Cost and reserve have a proportional (almost linear) relationship.

\section{CONCLUSION}

The formulation and application of the $\mathcal{R}$-metric as a constraint in day-ahead ED is presented. Sensitivity analysis shows the relationship between major interacting variables and functions. The inverse dependence of the $\mathcal{R}$-metric with cost indicates that a higher level of $\mathcal{R}$ is preferable while minimizing the cost in an ED problem. Power flow constraints are not considered for showing applicability on a simple system. Larger and more complex systems with system constraints can be included in ED as future work. Multiple objectives such as load reduction can also be included in the ED in future.

\section{REFERENCES}

[1] M. Panwar, "Reliability quantification and visualization for electric microgrids," Master's thesis, Colorado State University, Fort Collins, Colorado, USA, 2012
[2] J. F. Prada and M. D. Ilic, "Pricing reliability: A probabilistic approach," Energetica, vol. 23, pp. 75-89, 1999.

[3] North American Electric Reliability Corporation (NERC), "AppendixF: Performance indexes and equations."

[4] M. Panwar, D. Zimmerle, and S. Suryanarayanan, "Data analysis and visualization for electric microgrids: A case study on the fortzed rdsi microgrid," in 2013 IEEE Green Technologies Conference (GreenTech), April 2013, pp. 330-337.

[5] M. Panwar, "Operation of electric microgrids under uncertainty," Ph.D. dissertation, Colorado State University, Fort Collins, Colorado, USA, 2017.

[6] S. Letendre, M. Makhyoun, and M. Taylor, "Predicting solar power production: Irradiance forecasting models, applications and future prospects," Solar Electric Power Association (SEPA), Washington, D.C., Tech. Rep., 2014.

[7] M. Panwar, S. Suryanarayanan, and R. Hovsapian, "A multi-criteria decision analysis-based approach for dispatch of electric microgrids," International Journal of Electrical Power and Energy Systems, vol. 88, pp. 99-107, 62017.

[8] Y. Han, P. Young, and D. Zimmerle, "Optimal selection of generators in a microgrid for fuel usage minimization," in 2013 IEEE Power Energy Society General Meeting, July 2013, pp. 1-5.

[9] U.S. Energy Information Administration, "Gasoline and diesel fuel update," accessed on Nov. 13, 2018. [Online]. Available: https://www.eia.gov/petroleum/gasdiesel/

[10] City of Fort Collins, "Time-of-Day (TOD) residential electric pricing," accessed on Nov. 12, 2018. [Online]. Available: https://is.gd/i9qFFc

[11] Platte River Power Authority, "Platte river projected loads," accessed on Nov. 12, 2018. [Online]. Available: https://www.prpa.org/load/load.htm

[12] Colorado State University, "Fort Collins weather station data access," accessed on Nov. 12, 2018. [Online]. Available: https://is.gd/17wdB0

[13] W.-T. Huang, K.-C. Yao, and C.-C. Wu, "Using the direct search method for optimal dispatch of distributed generation in a mediumvoltage microgrid," Energies, vol. 7, no. 12, pp. 8355-8373, 62014. 